May 15, 2002

\title{
The Physical Properties of a Flaring Loop
}

\author{
S. M. White ${ }^{1}$, M. R. Kundu ${ }^{1}$, V. I. Garaimov ${ }^{1}$, T. Yokoyama $^{2}$ and J. Sato ${ }^{2}$ \\ ${ }^{1}$ Astronomy Department, University of Maryland, College Park, MD 20742 \\ ${ }^{2}$ Nobeyama Radio Observatory, National Astronomical Observatory of Japan, Minamisaku, \\ Nagano 384-13, Japan
}

\begin{abstract}
We use high-resolution radio observations to study the physical parameters of a flaring loop. The loop is visible at radio wavelengths due to gyrosynchrotron emission by nonthermal electrons (energies typically above several hundred $\mathrm{keV}$ ) accelerated by the flare. We are able to measure the loop thickness and length with a precision of order $1^{\prime \prime}$. We find that the loop length increases from about $60^{\prime \prime}$ initially to about $80^{\prime \prime}$ in the decay phase of the event. The loop (averaged along its length) initially is no more than $3^{\prime \prime}$ wide. The soft and hard X-ray data obtained with the SXT and HXT telescopes on the Yohkoh satellite are consistent with the same loop as observed at radio wavelengths (although the soft X-ray morphology has some small differences early in the event). This event was accompanied by a coronal mass ejection and a coronal dimming visible in SOHO/EIT images, so it involved a very large volume of the corona, yet the radio observations clearly indicate that much of the energy release in the low corona was restricted to a region apparently no more than $2000 \mathrm{~km}$ across. As the event proceeds the loop develops a bright feature at the looptop in both the radio and soft X-ray images that cannot be reproduced in gyrosynchrotron loop models in which the electron distribution has relaxed by pitch angle scattering to fill the loop. This prevents us from using the flare properties to measure the magnetic field strength and variation along the loop. The bright looptop source may require that trapping of electrons take place at the looptop late in the event.
\end{abstract}

Subject headings: Sun: flares - Sun: corona - Sun: radio radiation

\section{INTRODUCTION}

Observations of distinct magnetic loops are one of the major research tools for understanding conditions in the solar corona. Because the corona is collisionless the transport of physical 
properties across field lines (as distinct from transport by motions of the field lines themselves) is very slow (i.e., the timescale for transport orthogonal to the field is much longer than other relevant timescales such as propagation of plasma along the field and Alfvèn wave transit times), and the assumption that transport is predominantly along field lines, combined with the conservation of magnetic flux, allows us to constrain some physical parameters that cannot be observed. EUV and soft X-ray observations of distinct loops can be used to infer temperatures and thus to study the dependence of physical parameters with height in the corona. Studies such as these have been used to test theories of coronal heating. Loops could in principle be used to infer magnetic field strengths in the solar corona by using the observed change in the cross section of a loop with height in conjunction with a measurement of the field strength at the loop footpoint in the photosphere (e.g., Klimchuk et al. 1992; Aschwanden 1998), although in practice it is not straightforward to identify the magnetic field at a footpoint.

Radio observations of coronal loops offer a quite different viewpoint. In particular, loops rendered visible by the gyrosynchrotron emission from nonthermal electrons are particularly valuable for the study of magnetic fields in the corona because the emission mechanism itself depends intrinsically on the magnetic field in the loop, and the emission mechanism is well understood. Gyrosynchrotron loops have projection effects that are very different from those exhibited by optically-thin bremsstrahlung-emitting loops. The dependence of loop appearance on physical parameters has been investigated by a number of groups (e.g., Petrosian 1982; Dulk \& Dennis 1982; Klein \& Trottet 1984; Alissandrakis \& Preka-Papadema 1984; Preka-Papadema \& Alissandrakis 1988, 1992; Preka-Papadema et al. 1997; Bastian et al. 1998; Nindos et al. 2000; Kundu et al. 2001b). However, only rarely have the observations been of sufficient quality to be used to exploit this understanding. Seldom have complete loops been seen in the data. More typically, loop footpoints are seen (Shevgaonkar \& Kundu 1985; Bastian \& Kiplinger 1991; Alissandrakis et al. 1993; Kundu et al. 1995; Nishio et al. 1997) or, at low frequencies, a portion of the looptop is optically thick and dominates the emission (Marsh \& Hurford 1980; Marsh et al. 1981; Holman et al. 1982; Shevgaonkar \& Kundu 1985; Bastian \& Kiplinger 1991). Gary et al. (1996) observed a long-lived, large, complete flare loop but the emission mechanism was thermal bremsstrahlung and less information on the magnetic field structure was available.

More recently there have been several examples of flare loops suitable for direct comparison with models. Nindos et al. (2000) observed a flare with the Very Large Array in which the flare loop was visible at $5 \mathrm{GHz}$ and simultaneously the loop footpoints were imaged at $15 \mathrm{GHz}$. They were able to show that the magnetic field in the loop varied from $870 \mathrm{G}$ at the footpoint to $280 \mathrm{G}$ at the looptop, $1.9 \times 10^{9} \mathrm{~cm}$ above the surface. By contrast, Kundu et al. (2001b) observed 3 more flares with the Nobeyama Radioheliograph at 17 and $34 \mathrm{GHz}$ in which complete loops were found, and showed that the lack of variation in brightness along the loop required that there be little magnetic field variation from footpoint to looptop. This is consistent with studies of nonflaring loops in the 
EUV and soft X-rays which show little change in the apparent thickness of loops from footpoint to looptop (Klimchuk 2000; Watko \& Klimchuk 2000). We note however that selection of events in which a complete loop is visible at high radio frequencies necessarily selects in favor of loops with relatively little variation of $\mathrm{B}$ along the loop.

It requires radio data of exceptional quality in order to be able to measure important parameters such as loop length as a function of time and loop thickness as a function of distance along the loop. In this paper we take advantage of such data obtained with the Nobeyama Radioheliograph to study the physical conditions in a flare loop and compare them with models and with X-ray images from the Yohkoh spacecraft. The radio emission is produced by nonthermal electrons accelerated by the flare, and thus the properties of the loop reveal something about the acceleration region itself. We discuss the observations in the next section and then analyze the physical properties of the flare loop in the following section. We then conclude by comparing the measurements with loop models in order to address the physical properties of the loop.

\section{THE OBSERVATIONS}

We use data from the following sources to study this event: (i) imaging data at 17 and 34 $\mathrm{GHz}$ from the Nobeyama Radioheliograph (NoRH); (ii) radio light curves at 9.4, 17 and $35 \mathrm{GHz}$ from the Nobeyama Radio Observatory polarimeters (NoRP); (iii) hard X-ray images from the Hard X-ray Telescope (HXT) on the Yohkoh satellite; (iv) soft X-ray images from the Soft X-ray Telescope (SXT) on Yohkoh; (v) extreme ultraviolet images in the Fe XII $195 \AA$ line from the Extreme-ultraviolet Imaging Telescope (EIT) on the SOHO satellite (vi) and longitudinal magnetograms from the Michelson Doppler Interferometer (MDI) on SOHO. Since the data sets and data processing are very similar to previous event analyses we will not provide details of them here; the analyses are described in, e.g., Kundu et al. (2001a).

\subsection{Time Profiles}

Figure 1 shows radio and X-ray time profiles of this event. It occurred at heliographic coordinates S23E60 at 03:06 UT on 1999 May 29. The figure shows 9.4, 17 and $35 \mathrm{GHz}$ time profiles at 1 second time resolution; time profiles in all four HXT channels (LO: $14-23 \mathrm{keV}, \mathrm{M1}$ : 23-33 keV, M2: 33-53 keV, H: 53-93 keV) at varying resolutions of order of seconds; and the GOES 1-10 $\mathrm{keV}$ light curve at 3 second resolution (the GOES classification was M1.6).

The 14-23 keV hard X-ray data start to rise at 03:06:40 UT, reach a peak at 03:08:45 UT and then stay at a high level for almost 6 minutes before beginning a gradual decay. The channels with 
photons above $23 \mathrm{keV}$ show a similar rise but then decay much more rapidly, so that there is little emission detectable after 03:12 UT. The GOES light curve has a gradual rise that does not end until the HXT LO channel starts to decay.

The radio data do not resemble any of the X-ray light curves, with a rise starting at 03:08 UT, a relatively broad main peak lasting 3 minutes and then a slow decay phase continuing for another 20 minutes. The main radio peak has a radio spectrum falling with frequency above 9.4 $\mathrm{GHz}$, implying nonthermal emission from accelerated electrons in coronal magnetic fields as the emission mechanism (Dulk 1985; Bastian et al. 1998). Since the radio light curves do not resemble either the nonthermal hard X-ray light curve or the thermal soft X-ray light curve, we must attribute the radio emission to a population of energetic electrons that is not identical to those that produce the hard X-rays.

\subsection{Magnetic Context}

Figure 2 shows a SOHO/MDI magnetogram at the time of the flare (white contours) superimposed on a preflare SOHO/EIT $195 \AA$ Fe XII image and black contours showing the location of the flare loop in a post-flare SOHO/EIT $195 \AA$ Fe XII image. Since the three images used for this figure are all full-disk images, we estimate the accuracy of the overlays to be about one EIT pixel, or 2 " 6 . Because the flare occurred close to the limb, the magnetic field may show false inversions on the limbward side of sunspots due to the canopy effect.

The flare occurred in a region containing positive-polarity spots, and the location of the southern footpoint of the flare loop appears to be very close to one of these spots. The northern footpoint, however, lies above a region of much weaker mixed-polarity field. On this basis we would expect that the flare loop will show stronger magnetic field strengths at its southern footpoint, while precipitation of any trapped nonthermal electrons will occur preferentially at the weaker-field northern footpoint, which may thus show stronger hard X-ray emission.

This event was also associated with a CME and with a coronal dimming event. Figure 3 presents half-resolution EIT $195 \AA$ Fe XII images taken just before and just after the flare, and their difference image. There was an apparent evacuation of the corona, or at least a density depletion, in the volume apparently lying above the main flare loop in projection. It is also possible that the density depletion lay at longitudes eastward of the flare loop and appears above the flare loop due solely to projection effects. The coronal dimming could be a temperature effect rather than a density depletion if the material affected is heated so that it no longer radiates in the $195 \AA$ bandpass, but one then expects this material to show up as bright at other wavelengths, which apparently is not seen, and one requires a mechanism to heat nearly instantaneously a very large 
volume of the corona. Despite the influence of this event on a large volume as implied by the $\mathrm{CME}$ and coronal dimming, we will see that the flare energy release visible in soft X-rays and nonthermal electrons was confined to a narrow bundle of magnetic field lines.

\subsection{Loop Images}

Figure 4 presents radio and soft X-ray images of this event which show that both the thermal and nonthermal emissions are dominated by a single loop. (To clarify this description, by the term "loop" we mean a collection of adjacent magnetic field lines sharing a common morphology and adjacent footpoints. Thus, a "single loop" does not mean a single field line; since magnetic field lines have no thickness there must be infinitely many field lines in a loop of finite volume and the field lines within the loop may have unresolved structure.) Each of the columns in this figure shows images at a certain wavelength: $34 \mathrm{GHz}$ total intensity images in the left column (there is no circular polarization information at $34 \mathrm{GHz}$ ), $17 \mathrm{GHz}$ total intensity images in the second column, contours of the degree of circular polarization at $17 \mathrm{GHz}$ in the third column, and Yohkoh/SXT images in the fourth column. The first radio images are earlier than the first SXT image because Yohkoh flare mode, which obtained full-resolution images adequate to resolve the loop, did not commence until 03:08. The radio images are produced using super-resolution techniques at the cost of some signal-to-noise (e.g., see the discussion in Nindos et al. 1999). The radio images in Fig. 4 are deconvolved using the CLEAN algorithm and the clean components are restored using "clean-beam" gaussians of 6" resolution at $34 \mathrm{GHz}$ and 9" at $17 \mathrm{GHz}$.

The $34 \mathrm{GHz}$ images (left column) initially show brightening in the two legs of what becomes the main flare loop. The loop top is initially faint but subsequently brightens, and by 03:11:46 the southern side of the loop top is the brightest feature at $34 \mathrm{GHz}$. Initially the southern leg of the loop is clearly brighter than the northern leg in the radio images, but the two legs of the loop show similar brightness levels throughout, and there is no brightness peak at the footpoints. The apparent height and footpoint separation of the loop are both of order $30^{\prime \prime}$.

The $17 \mathrm{GHz}$ total intensity images (second column) appear to be consistent with the $34 \mathrm{GHz}$ images being observed with poorer resolution, but with more flux (see Fig. 1). The degree of circular polarization at $17 \mathrm{GHz}$ is never very large, not exceeding 20-30\%, and while initially the largest circular polarization appears to be near the loop top or the northern leg, later in the flare it is

concentrated at the southern footpoint of the loop where (as we inferred above) the magnetic field is probably higher.

At first glance the SXT soft X-ray emission appears to be similar to the radio loop in shape and dimension, but on closer inspection some clear differences are evident. Initially the SXT emission 
appears to consist of two disjoint features: a shorter arc coincident with the southern leg of the radio loop, and a longer arc linking the northern footpoint of the radio loop and the loop top. As the event proceeds, the gap between these two features is filled, and the soft X-ray source takes on the appearance of a single loop brightest at its apex, as in the radio loops at later times.

Figure 5 shows expanded SXT images of the loop at a selection of times, with the shape of the radio loop overplotted. At early times the soft X-ray emission does not appear to come from a continuous loop. The radio images (and specifically those at $34 \mathrm{GHz}$ that have the highest spatial resolution) do appear to show a single continuous loop.

This flare was detected in hard X-rays by Yohkoh/HXT and images can be made in all 4 energy channels but with varying time resolutions. Figure 6 compares HXT images at selected times with a representative SXT image. In the rise phase before 03:09 UT (left column) all the HXT channels show sources at both of the inferred footpoints of the flare loop, with the northern source being markedly stronger than the southern source. In the highest energy channel (53-93 keV) there are insufficient counts to make more than one good image, which is dominated by counts in the initial peak (Fig. 1). It shows that the highest energy emission is dominated by the weaker-field northern footpoint, as expected on the basis of the magnetic configuration (e.g., Melrose \& White 1979): the ratio of counts in the north to the south is at least 6 . The sequence of images in the LO channel shows a source appearing at the loop top and growing in strength to dominate the energy range 14-23 keV; there is no evidence for emission at the loop top in any of the higher-energy channels, so this event does not qualify as a "Masuda"-type event (Masuda et al. 1994).

An interesting feature shown by Figure 6 is that the southern footpoint of the loop moves westwards relative to the northern footpoint during the event. This can most clearly be seen by comparing the position of the southern footpoint in the HXT M1 and M2 images with the SXT image, which is the same in all panels. In the M1 images the southern footpoint moves about $8^{\prime \prime}$ in 5 minutes, or $19 \mathrm{~km} \mathrm{~s}^{-1}$ (in projection).

\section{LOOP PARAMETERS}

\subsection{Loop Fitting}

The radio images of the flare loop have sufficient dynamic range for us to determine most of the physical parameters in detail as a function of time. 128 radio images were made at both 17 and $34 \mathrm{GHz}$, from 03:06:01 UT to 03:20:01. The intervals between images are irregular but generally $5 \mathrm{~s}$, with a longer separation of $15 \mathrm{~s}$ for the first 7 and last 17 images.

For each image we first determined the loop shape (the "spine" of the loop) iteratively as 
follows. Given a point on the loop's spine, the next point is determined by stepping along the loop by a discrete amount in the direction specified by the previous two points. A Gaussian function is fitted to a one-dimensional cross-section of the intensity profile measured orthogonal to the loop direction. The location of the peak of the Gaussian is taken to be the loop position: this is appropriate because the true loop width must be smaller than the size of the clean beam used to restore the images $\left(6^{\prime \prime}\right.$ at $34 \mathrm{GHz}$ and $9^{\prime \prime}$ at $17 \mathrm{GHz}$; if this were not the case the loops would appear to have a FWHM much larger than that of the clean beam size). The width of the Gaussian is converted to the equivalent full width at half maximum (FWHM). The set of loop points resulting from this procedure is irregularly spaced and was converted to a regularly s paced grid for the final analysis. This fitting procedure occasionally had problems at the loop top where the loop curvature was sharp and the loop width appears to broaden considerably later in the event (Fig. 4).

To within the signal-to-noise, the 17 and $34 \mathrm{GHz}$ loop shapes resulting from this procedure were found to agree with each other. This is to be expected if both frequencies are produced by the same set of electrons. The radio spectrum (Fig. 1) falls with increasing frequency above $9 \mathrm{GHz}$, indicating optically-thin nonthermal emission at 17 and $34 \mathrm{GHz}$, and in this case the loop shapes should be the same at both frequencies. The fact that we find this to be the case can be taken to confirm the results of the fitting procedure. Representative examples of the fitted radio loop shape are overplotted on the soft X-ray images in Figure 5.

We note that the properties that we measure for this loop may not refer to the same set of magnetic field lines throughout the flare, so the interpretation of the evolution of the properties of the loop depends on the model being invoked (e.g., successive brightening of adjacent field lines versus a fixed set of field lines that moves and spreads apart).

\subsection{Loop Intensity Profiles}

Once the loop shapes have been determined, we can plot the variation of intensity with distance along the loop. Again, since the true loop width is smaller than the nominal resolution of the images, we can simply use the intensity of the radio image at the peak location for this purpose. This is shown in Figure 7 for $34 \mathrm{GHz}$ total intensity, and $17 \mathrm{GHz}$ total intensity and circularly polarized flux. Time increases from the bottom to the top of the figure. For convenience, the loop is extended beyond the point at which the loop cross-section can reliably be fitted at both ends by linear projection of the last few reliable points. This is a crude movie-like representation of the evolution of the loop with time.

The $34 \mathrm{GHz}$ loops initially show peaks in the two legs of the flux tube, but as is apparent in Fig. 4 the intensity profiles do not show a sharp peak at the footpoints: rather, it appears that an 
extended region of each leg above the footpoint is bright. As time proceeds, the peaks in the legs are replaced by a peak in the center, with the northern leg becoming brighter than the southern leg.

At $17 \mathrm{GHz}$ the signal-to-noise is somewhat better and the resolution is somewhat poorer, so the intensity profiles are smoother than at $34 \mathrm{GHz}$. They show essentially the same behaviour, but with the central peak appearing prominent slightly earlier than at $34 \mathrm{GHz}$. We do not believe that there is a significant difference between the two frequencies in this respect. As noted earlier, the polarization of this event is weak, but the southern footpoint shows a peak throughout. There appears to be significant polarization at the northern footpoint in the early stages of the event. Weak polarization is expected from optically thin gyrosynchrotron emission whenever the source is viewed along a line of sight that is nearly orthogonal to the magnetic field direction in the source, which may well be the case for the footpoints of this loop, so the weak polarization is not surprising.

\subsection{Loop Length}

A clear feature of Fig. 7 is that the length of the loop increases with time. Defining the ends of the loop at each time by the points at which the flux drops to $10 \%$ of the maximum intensity, we can measure this effect and the results are shown in Fig. 8. Both 17 and $34 \mathrm{GHz}$ lengths are shown. The difference between the two frequencies is a consequence of the definition of the loop length and the different signal-to-noise in the images, so the absolute length measurements have little meaning but the variation is similar at both frequencies until late in the event when the $34 \mathrm{GHz}$ emission is weak and the measurements are less significant. The $17 \mathrm{GHz}$ data show the loop length increasing by about $20^{\prime \prime}$ from 03:08 to 03:20 UT. The corresponding velocity is $19 \mathrm{~km} \mathrm{~s}^{-1}$, which is similar to the velocity inferred for the motion of the hard X-ray source at the southern footpoint. The radio images also seem to show elongation of the southern leg relative to the northern leg during the event (Fig. 4). Since this is a CME event, it is possible that the footpoint separation is similar to the well-known spreading of the $\mathrm{H} \alpha$ ribbons in a two-ribbon flare. Typical velocities for this phenomenon are $5-20 \mathrm{~km} \mathrm{~s}^{-1}$ (Zirin 1988). Sakao (1999) reports that the hard X-ray footpoints of loops typically separate at (deprojected) speeds of order $50 \mathrm{~km} \mathrm{~s}^{-1}$, which is similar to the projected separation speed measured here. However, Figures 2 and 3 suggest that the loop is predominantly north-south in orientation, with the southern footpoint to the east of the northern

footpoint. In this geometry, spreading of the footpoints is not likely to produce the observed result in which the southern leg lengthens and the southern footpoint moves to the west. 


\subsection{Loop Width}

The loop fitting procedure employed here also yields the variation of loop thickness with distance along the loop. Figure 9 shows the full width at half maximum of the Gaussian fits to loops for periods when the signal to noise is adequate to make a measurement along most of the loop. We use $34 \mathrm{GHz}$ loops since those images have better spatial resolution, but by 03:12 the 34 $\mathrm{GHz}$ emission is too weak for this to be done. Figure 9 indicates that the width was essentially constant along the loop except near the top of the loop where, as noted earlier, the loop curvature complicates the fitting of the loop thickness, and in addition the loop broadens at its top as the flare proceeds in both radio and X-ray images.

We use the median of the widths of the loop at each point along the loop to represent the overall loop width. There is little difference between the median of these points and the mean. The results are plotted as filled circles in Figure 10. The width appears to be larger at $17 \mathrm{GHz}$ than at $34 \mathrm{GHZ}$, but the resolution of the images at the two frequencies is different: the $17 \mathrm{GHz}$ images have been convolved with a $9^{\prime \prime}$ (FWHM) Gaussian in restoring the clean components while the 34 $\mathrm{GHz}$ images have been convolved with a $6^{\prime \prime}$ Gaussian. To determine the true width of the loop we need to deconvolve the size of the gaussian functions used to smooth the images from the raw measured widths. The results are shown as open circles on Figure 10. The deconvolved 17 and $34 \mathrm{GHz}$ widths agree with each other remarkably well considering the difference between the raw measurements at the two frequencies. Both show the mean loop width increasing from about $3^{\prime \prime}$ initially to about $6^{\prime \prime}$ by 03:12 UT, when the radio loops are dominated by the looptop source and the mean width no longer has much meaning.

To check that these widths are plausible and independent of the deconvolution method, we have produced super-resolved images at selected times using maximum entropy deconvolution of images with a pixel size of 0. " 5 . The maximum entropy method utilizes a smoothness constraint, i.e., the result of the deconvolution is the smoothest image that is consistent with the dirty map and the dirty beam to within the specified noise level. Since the noise level in the images is independent of pixel size whereas the signal per pixel does vary with pixel size, effectively we are sacrificing dynamic range to achieve high spatial resolution. The results are shown in Figure 11. In these images the effective spatial resolution varies across the image depending on the local signal-tonoise level, so it is not straightforward to state what the spatial resolution is at any given point. However, at 03:09 UT the images appear to be consistent with a median width in the brightest locations of order $3^{\prime \prime}$, as determined from Figure 9. It cannot be ruled out that the true loop width is less than this initially, but the images clearly show that the loop width increases with time as shown in Figure 10, and so the loop width must be of order $5^{\prime \prime}$ or more at later stages. 


\section{FLARE LOOP MODELS}

\subsection{Radio Loop Models}

The radio data for this event are of such quality that the physical parameters determined for the flare loop are amongst the best measurements yet for a flare loop observed at any wavelength. The results of the previous section indicate that the flare loop during the impulsive phase was approximately $60^{\prime \prime}$ long in projection and just $3^{\prime \prime}$ across. The corresponding mean brightness temperatures are of order $5 \times 10^{7} \mathrm{~K}$ at $17 \mathrm{GHz}$ (flux $200 \mathrm{sfu}$ ) and $5 \times 10^{6} \mathrm{~K}$ at $34 \mathrm{GHz}$ (peak flux $80 \mathrm{sfu})$.

As noted earlier, the radio spectrum indicates that the loop radiates via nonthermal gyrosynchrotron emission, i.e., mildly relativistic electrons gyrating in a coronal magnetic field of order hundreds of gauss. The emissivity of this mechanism has a strong dependence on magnetic field strength and on the angle between the viewer's line of sight and the magnetic field direction in the source: the emissivity is much larger when this angle is close to $90^{\circ}$ than when the source is viewed along the magnetic field. It should be noted that because of the viewing-angle dependence of gyrosynchrotron emission, loops emitting by this mechanism have projection effects very different from those of soft X-ray loops: in particular, whereas looking along a loop produces bright emission in X-rays due to the increased line-of-sight column, emission from a gyrosynchrotron loop actually decreases because the low opacity parallel to the line of sight has a stronger effect than the increase in column depth.

Since 17 and $34 \mathrm{GHz}$ are clearly on the high-frequency side of the radio spectral peak in this event, their source must be optically thin. Unfortunately, the loop intensity profiles are not consistent with standard filled-loop models of such sources for most of the event. As has been shown by many previous studies, an optically thin loop in which the magnetic field declines from the footpoint to the looptop will generally be brightest at the footpoints of the loop. The angle between the magnetic field and the line of sight also plays an important role, but for this loop this angle is expected to be large almost everywhere along the loop if the loop is indeed north-south as appears to be the case (e.g., Fig. 2). Even if the loop has a significant east-west orientation the looptop emission will be diminished since an east-west loop near the limb has a relatively low angle between the magnetic field direction at the top of the loop and the line of sight while the legs have a large viewing angle, which should further enhance the ratio of footpoint to looptop brightness. Figure 12 shows model flare loops calculated using an inhomogeneous gyrosynchrotron code with realistic parameters and a geometry similar to the loop observed in this event, for a very optically thick frequency $(5.0 \mathrm{GHz})$, a marginally optically thick frequency $(9.4 \mathrm{GHz})$ and an optically thin frequency $(17 \mathrm{GHz})$. At the optically thick frequency the sides of the loop close to the loop top are bright while the footpoints are relatively faint; at the higher optically thin frequency the 
footpoints are bright and the level of emission decreases monotonically as height increases. The optically thick frequency is completely unpolarized at the footpoints of the loop where the optical depth is high, and moderately polarized at the loop top where the loop is viewed nearly along the line of sight and the loop is only marginally optically thick (optical depth close to unity for this calculation); at the optically thin frequency there is only weak polarization at the footpoints because the magnetic field is nearly orthogonal to the line of sight.

Note that the observed loop profiles for this event resemble the optically thin limit in Figure 12 in the early phase of the event when the two legs of the loop are bright and the looptop is faint, but none of the model loops resemble the observed source in the later stages of the event when the looptop dominates both the radio and X-ray emission.

If the magnetic field is constant along the loop then (since it follows that the electron distribution will be homogeneous on timescales longer than the bounce time, of order 1 second or less) the relative brightness of looptop and footpoints is solely determined by the viewing angle. One could then perhaps produce a looptop radio source if both legs of the loop point at the observer whereas the looptop is orthogonal to the line of sight, but given the projected shape of the loop and its location so close to the limb the resulting deprojected shape would be very non-radial and highly elongated along the line of sight, with narrow footpoint separation but very long legs. Such a morphology is not plausible. In an optically-thin gyrosynchrotron model for a loop with the apparent geometry of this event, it is not possible for the looptop to appear as bright as it does late in the event if the loop is filled with energetic electrons along its length. Further, the change in the appearance of the loop with time has no obvious explanation. However, if the energetic electrons were initially distributed throughout the loop but later were trapped preferentially in a low-field region near the top of the loop and precipitated only slowly, then initially the whole loop would be seen and while at later stages there would be few electrons at the loop footpoints and the radio emission could be dominated by the looptop source.

\subsection{Comparison with X-ray Loop Models}

In this event there is no strong evidence for a looptop hard X-ray source at energies corresponding to the M1 channel or higher; the detection of emission in the HXT M1 image from 03:09:18 to 03:09:49 is marginal at best. However, there is a strong looptop source evident in the HXT L channel as well as in the SXT images (Fig. 6), but only later in the event. We believe that this fact rules out projection effects as the cause of the late brightening at the looptop: if the brightening is a projection effect then it should have been seen earlier in the event as well, unless the looptop elongated dramatically exactly in the direction pointing at the telescope, which we submit is implausible. The geometry of the event (Fig. 3) also suggests a north-south loop, rather 
than an east-west loop in which projection effects would be expected. If the looptop brightening is not due to projection effects, we must look for another cause. The production of hard X-rays by bremsstrahlung is proportional to the product of the densities of the ambient thermal electrons and the nonthermal electrons. In order to have a looptop source other than by projection effects, one of these densities must peak at the looptop. Hydrodynamics does not allow a situation in which the dominant ambient thermal density at the looptop is significantly higher than in the legs of the loop to be sustained for more than tens of seconds (the looptop sources then have much higher pressure than the rest of the loop and should expand at about the proton thermal speed of $400 \mathrm{~km} \mathrm{~s}^{-1}$ at 2 $\left.\times 10^{7} \mathrm{~K}\right)$. A hard X-ray source confined to the looptop therefore seems to require trapping of the nonthermal electrons there, perhaps due to a combination of large pitch angles for the nonthermal electrons and a strong gradient in magnetic field strength from the top of the loop to the bottom in order for magnetic mirroring to be effective (Fletcher \& Martens 1998; Metcalf \& Alexander 1999). Scattering due to wave turbulence localized at the top of the loop may also play a role in trapping energetic electrons there (Petrosian \& Donaghy 1999). As in this event, many flares exhibit soft X-ray kernels which are observed to occur at looptops for extended periods, and unless they are all due to projection effects, they imply the persistence of substantial pressure excesses at the looptop despite the difficulties in explaining them described above (Feldman et al. 1994; Doschek et al. 1995). Such high-density sources have also been invoked to explain the hard X-ray looptop sources (Wheatland \& Melrose 1995).

We note that the looptop radio source is only a factor of 2 brighter than the legs of the loop late in the event, whereas in soft X-rays the looptop source is an order of magnitude brighter than the lower portions of the loop legs. This could also be consistent with a loop model in which the legs have higher magnetic field than the looptop but the electrons are preferentially trapped in the low-field region at the looptop: the electrons which do make it to the loop legs will experience a higher magnetic field and radiate more efficiently at radio wavelengths, whereas the soft X-ray emission relies on density which need not be higher in the loop legs.

\subsection{Implications for Energy Release Models}

In one model for eruptive flares the flare loop is formed by the reconnection of previously open field lines on opposite sides of a helmet streamer which are brought together in a Y-type neutral current sheet (known as the "CSHKP" model: Carmichael 1964; Sturrock 1968; Hirayama 1974; Kopp \& Pneuman 1976). The early ideas have been further developed, e.g., Tsuneta (1996) and Shibata $(1996,1999)$ and references therein. In this model the loop consists of successively higher field lines created by reconnection at a site which moves upwards in the corona as time progresses. Thus the lower (innermost) field lines of the flare loop are fixed while the upper edge of the flare 
loop expands with time as higher and higher closed field lines are created. The hard X-rays will occur predominantly at the footpoints of the most recently reconnected field lines and thus when both footpoints are visible they should be seen to separate with time: this is often observed to be the case (Sakao 1999). This phenomenon might be present in this event, although the images give the impression that only the southern footpoint shows motion and only the southern leg appears to lengthen (cf. Fig. 6 and the discussion in subsection 3.3). In this scenario we expect to see the thickness of the bundle of illuminated field lines growing with time as more closed field lines are created by reconnection, and the trend seen in Figure 10 is consistent with this idea. The expansion in width is only $3^{\prime \prime}$ over 5 minutes, i.e., much smaller than the motion of the southern footpoint. We also expect to see the legs move apart. The north-south separation of the spines in the two legs of the loop appears to increase by about $4^{\prime \prime}$ from 03:08 to 03:10, but thereafter does not change. If the basic CSHKP model is relevant for this event, it is peculiar that the fixed footpoint occurs in the weaker magnetic field region while the faster moving footpoint lies over much stronger magnetic fields: equal amounts of flux must reconnect at the current sheet and this would seem to require that the weaker-field footpoint show greater apparent motion because it has to move further to sweep up the same amount of magnetic flux as the footpoint in the stronger-field region.

\section{CONCLUSIONS}

We have used high-resolution radio observations to study the physical parameters of a flaring loop. The loop is visible at radio wavelengths due to gyrosynchrotron emission by nonthermal electrons (energies typically above $500 \mathrm{keV}$ ) accelerated by the flare. The data are of sufficient quality that we are able to measure the loop thickness and length with a precision of order $1^{\prime \prime}$. Since the radio spectrum indicates that the 17 and $34 \mathrm{GHz}$ emission must be optically thin, we can use consistency between the observations at 17 and $34 \mathrm{GHz}$ to confirm the measurements. We show that the loop length increases from about $60^{\prime \prime}$ initially to about $80^{\prime \prime}$ in the decay phase of the event. The loop (averaged along its length) is measured to be just $3^{\prime \prime}$ wide initially, increasing to at least 6 "later on.

The soft and hard X-ray data obtained with the SXT and HXT telescopes on the Yohkoh satellite are consistent with the same set of field lines as illuminated at radio wavelengths (although the soft X-ray morphology has some small differences early in the event). This event was accompanied by a coronal mass ejection and a coronal dimming visible in SOHO/EIT images, so it involved a very large volume of the corona, yet the radio observations clearly indicate that much of the energy release in the low corona was restricted to a region no more than $2000 \mathrm{~km}$ in the transverse dimension. This is a challenge for our understanding of the relationship between the large-scale aspects of coronal mass ejections and their associated effects in the low corona. 
Unfortunately, the loop morphology is not consistent with standard gyrosynchrotron models for flare loops and we are unable to determine the magnetic field strength and the variation of B along the loop by comparison with model loops as we have done in other events (Nindos et al. 2000; Kundu et al. 2001b). As the event proceeds the loop develops a bright feature at the looptop in both the radio and soft X-ray images. Such a feature cannot be reproduced in gyrosynchrotron loop models in which the electron distribution has relaxed by pitch angle scattering to fill the loop: in such models the footpoints would be expected to be brighter than the looptop at the observed frequencies, and this is observed early in the event. The bright looptop source may require that trapping of electrons take place at the looptop later in the event. The soft X-ray brightening there requires that the density also be high at that location.

M. R. Kundu wishes to thank the staff of NAOJ and NRO (Japan) for their help and hospitality during his stay at the observatories. This research at Maryland was supported by NSF grants ATM 99-90809 and INT-98-19917, and NASA grant NAG 5-8192. We gratefully acknowledge the opendata policies of the ISAS/NASA satellite Yohkoh and the ESA/NASA satellite SOHO and their instrument teams which make multi-wavelength research such as this much easier. We thank the referee for urging us to think carefully about the assumptions made in this analysis. 


\section{REFERENCES}

Alissandrakis, C. E., Nindos, A., \& Kundu, M. R. 1993, Solar Phys., 147, 343

Alissandrakis, C. E. \& Preka-Papadema, P. 1984, Astron. Astrophys., 139, 507

Aschwanden, M. J. 1998, Astrophys. J., 502, 455

Bastian, T. S., Benz, A. O., \& Gary, D. E. 1998, Ann. Rev. Astron. Astrophys., 36, 131

Bastian, T. S. \& Kiplinger, A. 1991, in Max 91 Workshop No. 3 (Estes Park), ed. R. Winglee \& A. Kiplinger, 153

Carmichael, H. 1964, in AAS-NASA Symposium on Solar Flares, ed. W. N. Hess (NASA SP-50), 451

Doschek, G. A., Strong, K. T., \& Tsuneta, S. 1995, Astrophys. J., 440, 370

Dulk, G. A. 1985, Ann. Rev. Astron. Astrophys., 23, 169

Dulk, G. A. \& Dennis, B. R. 1982, Astrophys. J., 260, 875

Feldman, U., Hiei, E., Phillips, K. J. H., Brown, C. M., \& Lang, J. 1994, Astrophys. J., 421, 843

Fletcher, L. \& Martens, P. C. H. 1998, Astrophys. J., 505, 418

Gary, D. E., Wang, H., Nitta, N., \& Kosugi, T. 1996, Astrophys. J., 464, 965

Hirayama, T. 1974, Solar Phys., 34, 323

Holman, G. D., Kundu, M. R., \& Papadopoulos, K. 1982, Astrophys. J., 257, 354

Klein, K.-L. \& Trottet, G. 1984, Astron. Astrophys., 141, 67

Klimchuk, J. A. 2000, Solar Phys., 193, 53

Klimchuk, J. A., Lemen, J. R., Feldman, U., Tsuneta, S., \& Uchida, Y. 1992, Publ. Astron. Soc. Japan, 44, L181

Kopp, R. A. \& Pneuman, G. W. 1976, Solar Phys., 50, 85

Kundu, M. R., Grechnev, V. V., Garaimov, V. I., \& White, S. M. 2001a, Astrophys. J., 563, 389

Kundu, M. R., Nindos, A., White, S. M., \& Grechnev, V. V. 2001b, Astrophys. J., 557, 880 
Kundu, M. R., Nitta, N., White, S. M., Shibasaki, K., Enome, S., Sakao, T., Kosugi, T., \& Sakurai, T. 1995, Astrophys. J., 454, 522

Marsh, K. A. \& Hurford, G. J. 1980, Astrophys. J. (Lett.), 240, L111

Marsh, K. A., Hurford, G. J., Zirin, H., Dulk, G. A., Dennis, B. R., Frost, K. J., \& Orwig, L. E. 1981, Astrophys. J., 251, 797

Masuda, S., Kosugi, T., Hara, H., Tsuneta, S., \& Ogawara, Y. 1994, Nature, 371, 495

Melrose, D. B. \& White, S. M. 1979, Proc. Astron. Soc. Aust., 3, 369

Metcalf, T. R. \& Alexander, D. 1999, Astrophys. J., 522, 1108

Nindos, A., Kundu, M. R., White, S. M., Gary, D. E., Shibasaki, K., \& Dere, K. P. 1999, Astrophys. J., 527, 415

Nindos, A., White, S. M., Kundu, M. R., \& Gary, D. E. 2000, Astrophys. J., 533, 1053

Nishio, M., Yaji, K., Kosugi, T., Nakajima, H., \& Sakurai, T. 1997, Astrophys. J., 489, 976

Petrosian, V. 1982, Astrophys. J. (Lett.), 255, L85

Petrosian, V. \& Donaghy, T. Q. 1999, Astrophys. J., 527, 945

Preka-Papadema, P. \& Alissandrakis, C. E. 1988, Astron. Astrophys., 191, 365

-. 1992, Astron. Astrophys., 257, 307

Preka-Papadema, P., Alissandrakis, C. E., Dennis, B. R., \& Kundu, M. R. 1997, Solar Phys., 172, 233

Sakao, T. 1999, in Nobeyama Symposium on Solar Physics with Radio Observations, ed. T. S. Bastian, N. Gopalswamy, \& K. Shibasaki (Nobeyama Radio Observatory), 321

Shevgaonkar, R. K. \& Kundu, M. R. 1985, Astrophys. J., 292, 733

Shibata, K. 1996, Adv. Space Res., 17(4/5), 9

Shibata, K. 1999, in Nobeyama Symposium on Solar Physics with Radio Observations, ed. T. S. Bastian, N. Gopalswamy, \& K. Shibasaki (Nobeyama Radio Observatory), 381

Sturrock, P. A. 1968, in IAU Symp. 35: Structure and Development of Solar Active Regions, Vol. 35, 471 
Tsuneta, S. 1996, Astrophys. J., 456, 840

Watko, J. A. \& Klimchuk, J. A. 2000, Solar Phys., 193, 77

Wheatland, M. S. \& Melrose, D. B. 1995, Solar Phys., 158, 283

Zirin, H. 1988, Astrophysics of the Sun (Cambridge: Cambridge University Press) 


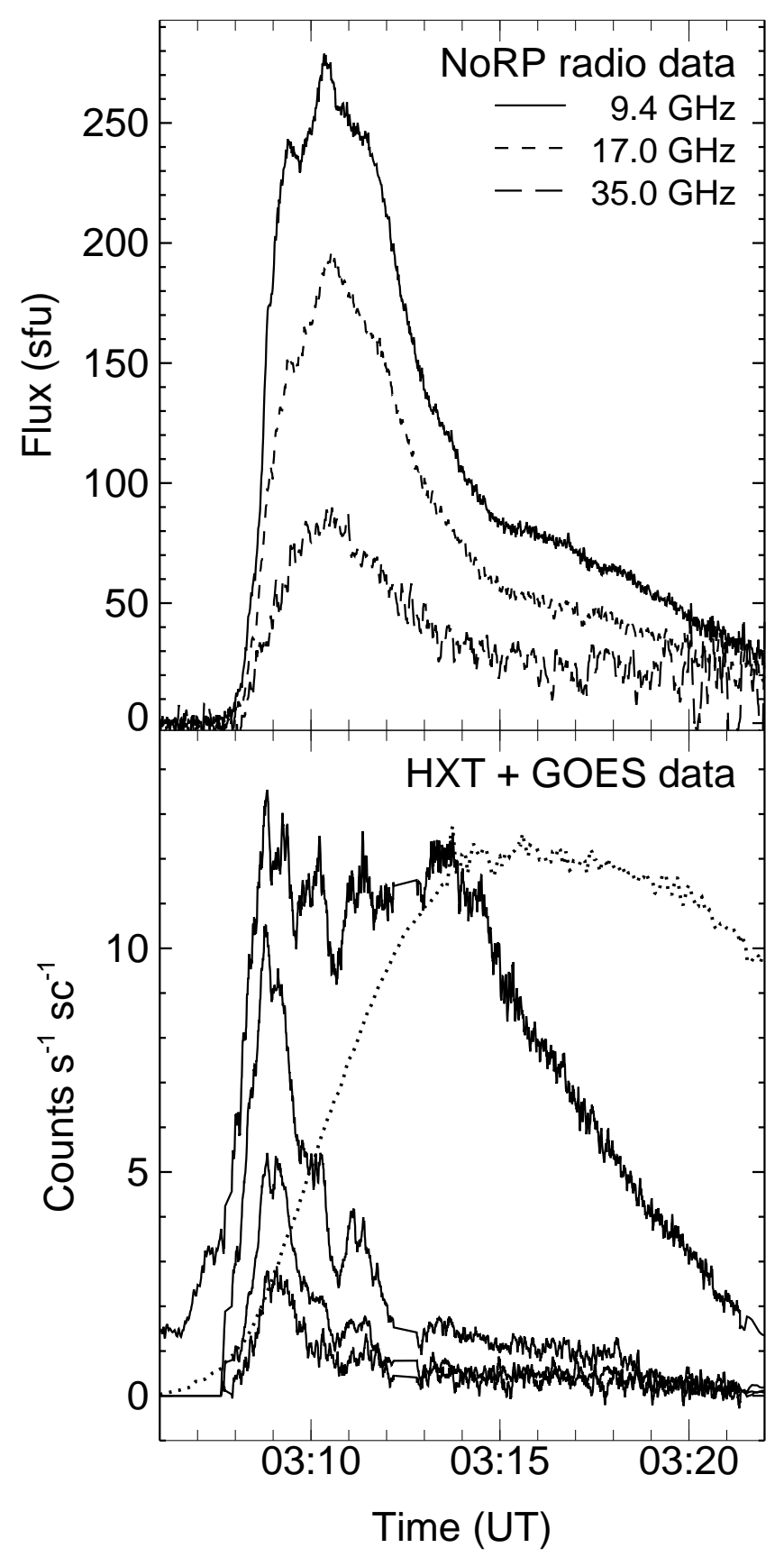

Fig. 1.- Lightcurves for the flare at radio (upper panel) and X-ray (lower panel) wavelengths. The upper panel shows data from the Nobeyama polarimeters at 9.4, 17 and $35 \mathrm{GHz}$ at 1 second resolution. The solid lines in the lower panel are the time profiles for the 4 Yohkoh/HXT channels (LO, 14-23 keV; M1, 23-33 keV; M2, 33-53 keV; and H, 53-93 keV) in order with the LO channel having the highest count rates and the $\mathrm{H}$ channel the lowest count rates. The dotted line shows the GOES 1.5-10 keV channel, scaled and preflare-subtracted; the peak rate is $1.5 \times 10^{-5} \mathrm{~W} \mathrm{~m}^{-2}$ (GOES class M1.5). 


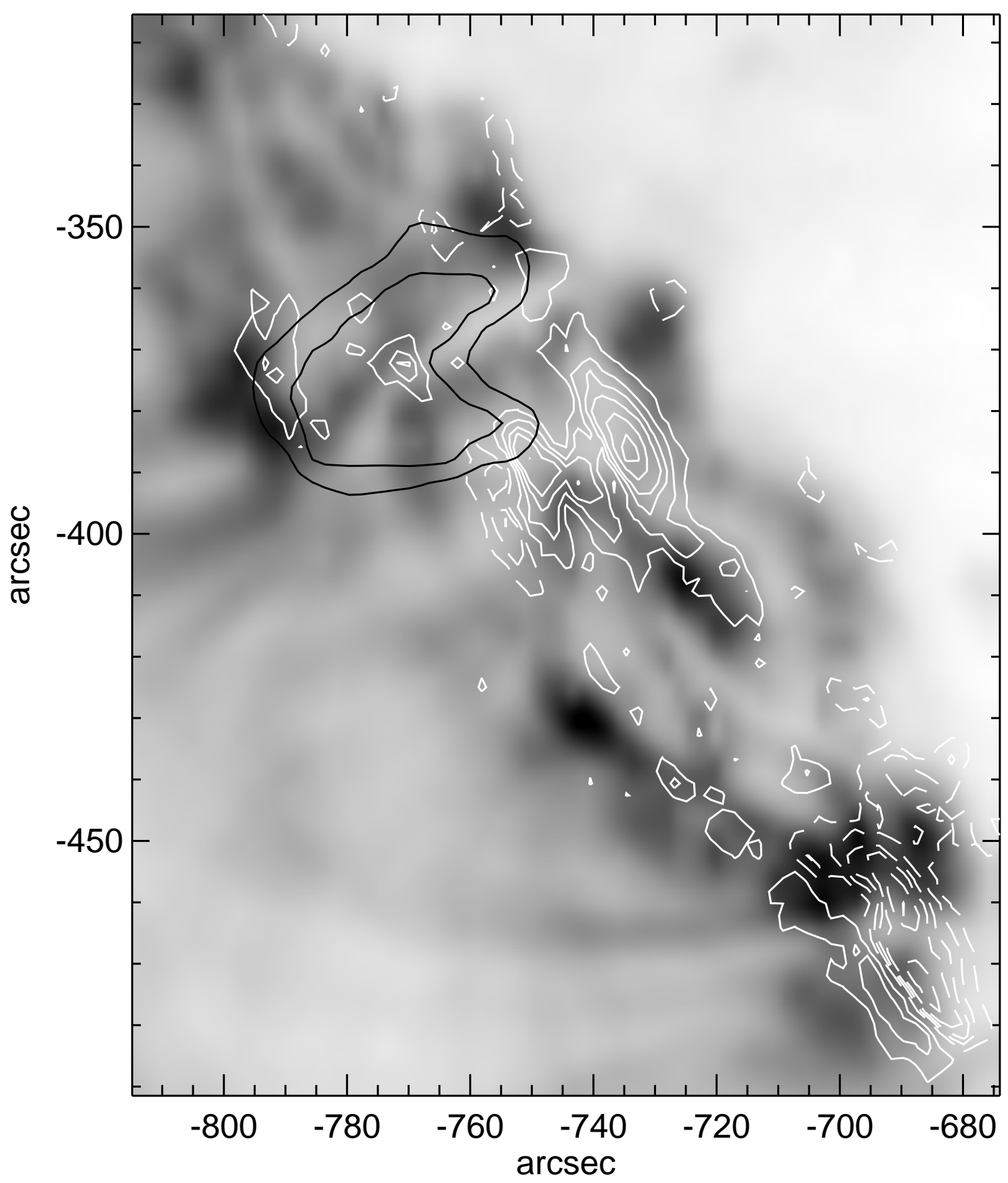

Fig. 2.- Overlay of (white) contours of a SOHO/MDI magnetogram from 03:12 UT on a greyscale representation of the full-resolution (2.6" pixels) SOHO/EIT $195 \AA$ Fe XII image from 01:13 UT rotated forward to the time of the magnetogram. Magnetic field contours are plotted at $\pm 100,200, \ldots, 800 \mathrm{G}$ (line of sight component) The black contours show the location of the flare loop in a half-resolution SOHO/EIT $195 \AA$ Fe XII image from 03:24 UT. 


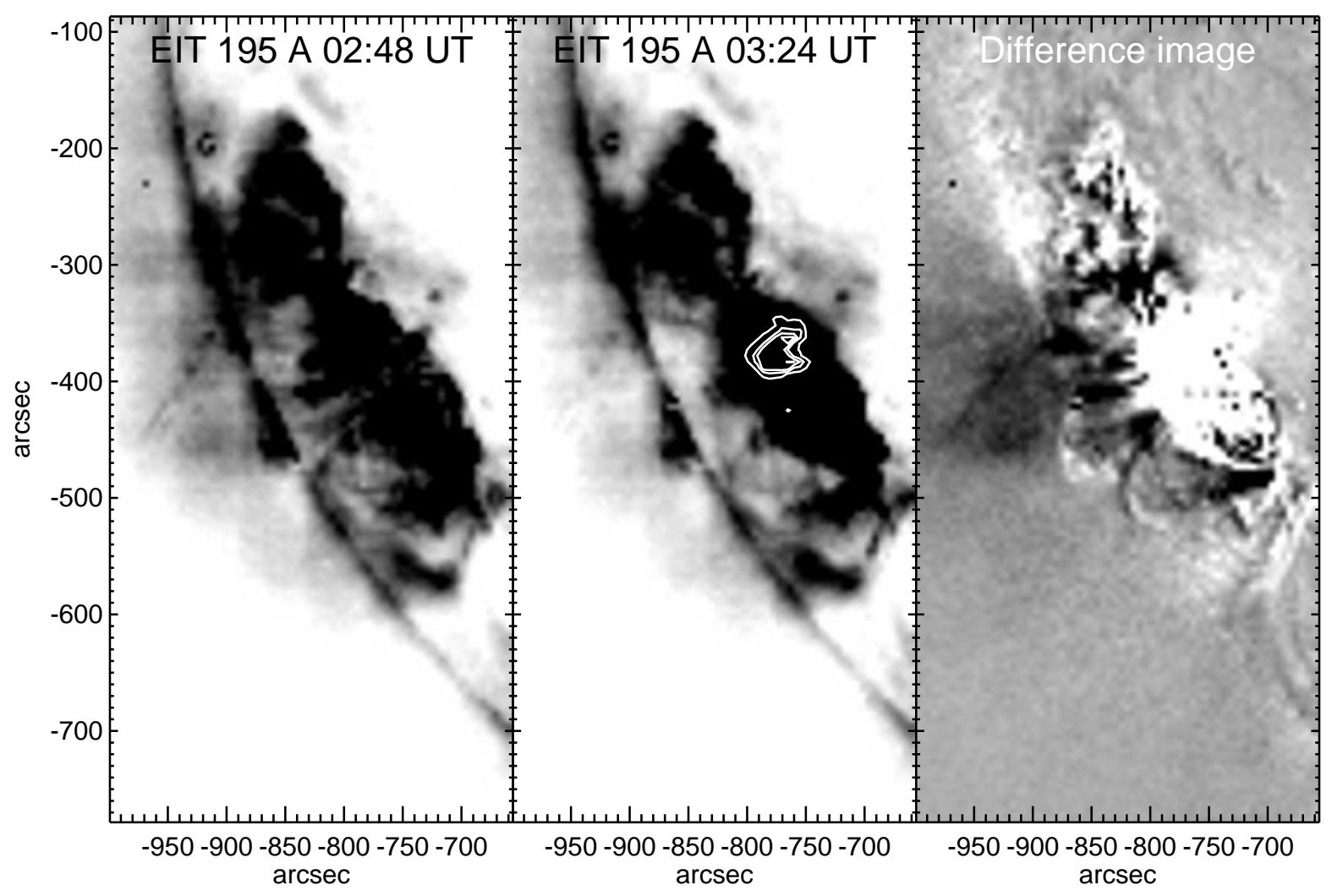

Fig. 3.- SOHO/EIT $195 \AA$ Fe XII half-resolution images (5!'2 pixels) before (left panel, 02:48 UT) and after (middle panel panel, 03:24 UT) the flare, and their difference image (right panel) showing the evacuation of a large volume above the flare site. The displays of the images are chosen to emphasize weaker features; the white contours in the middle panel show the location of the main flare loop in this image. 


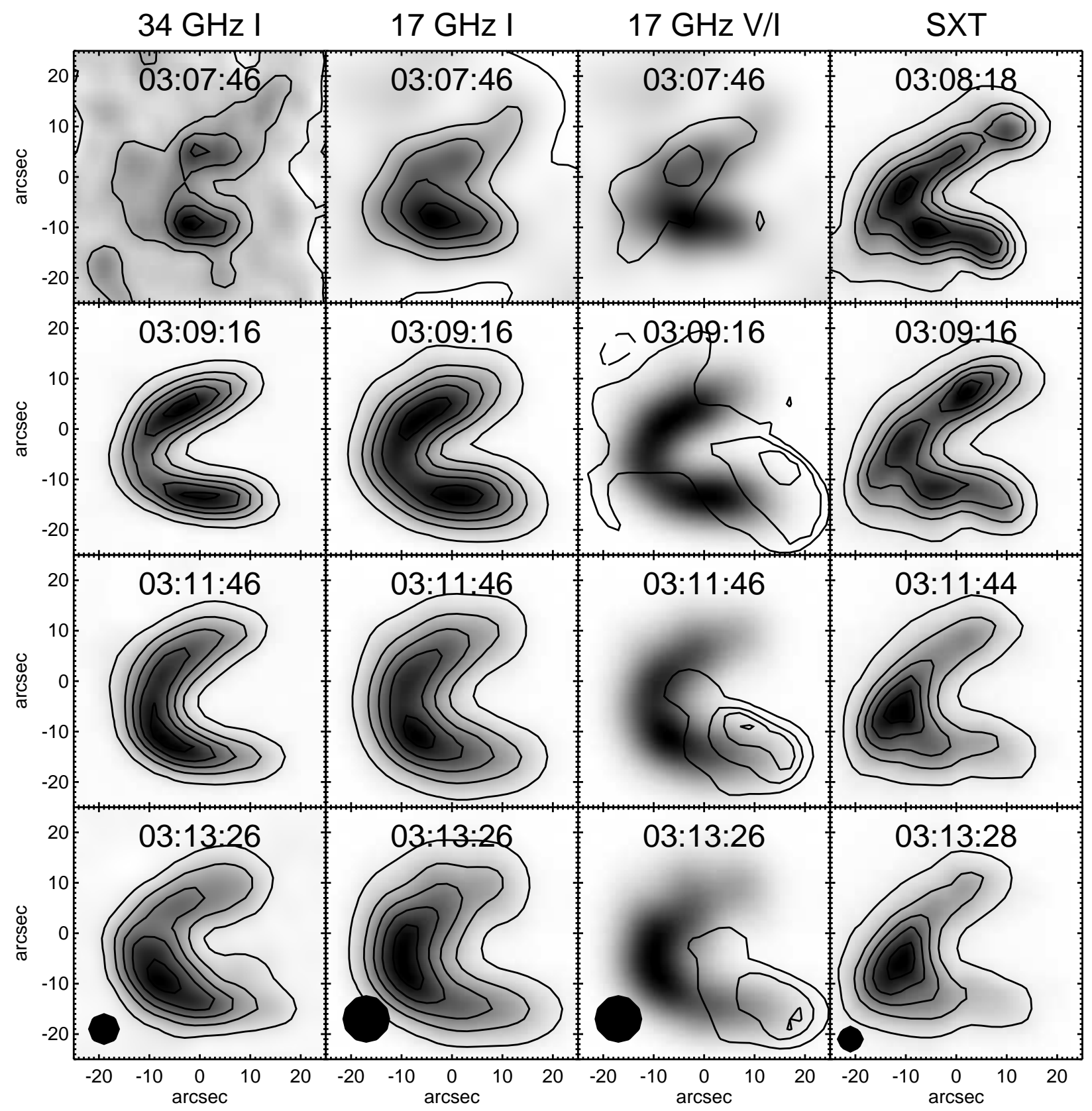

Fig. 4.- Images of the flare at radio and soft X-ray wavelengths at 4 times during the impulsive phase. The three left columns of radio images ( 34 and $17 \mathrm{GHz}$ total intensity with contours at 10 , $30, \ldots, 90 \%$ of the maximum, and contours of the $17 \mathrm{GHz}$ degree of circular polarization overlaid on greyscale images of the $17 \mathrm{GHz}$ total intensity) are obtained with the Nobeyama Radio Heliograph, while the right column contains full-resolution images from the Yohkoh/SXT telescope at times as close as possible to the times of the radio images (the first SXT image is later than the first radio images because SXT did not enter flare mode until 03:08; the SXT images use either the $\mathrm{AlMg}$ or Al12 filters). The resolutions of each type of image are shown in the bottom row of panels. Relative intensities at different times may be determined from Figure 1, with the SXT light curve being very similar to the GOES light curve. The degree of polarization contours are at $10 \%$ intervals. 


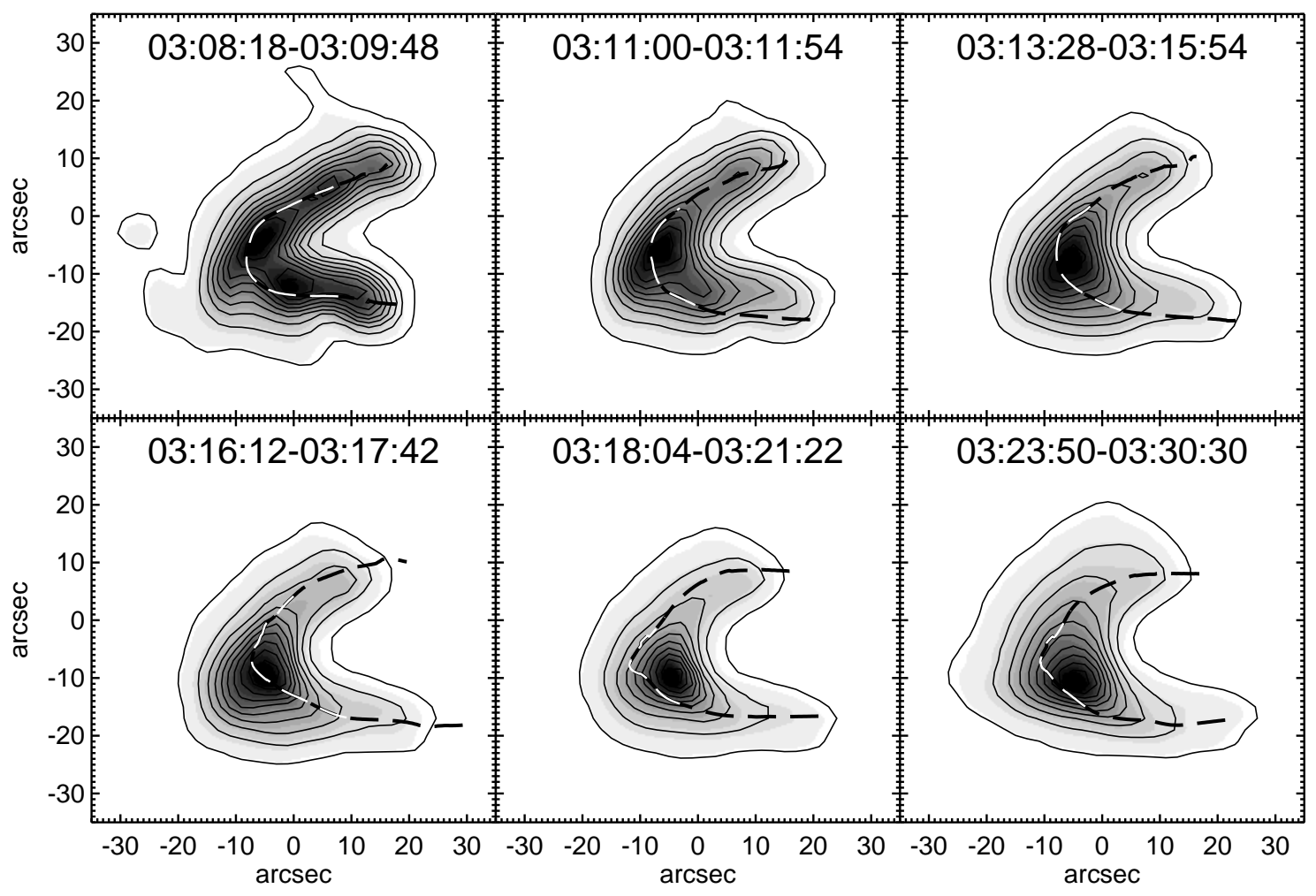

Fig. 5.- Yohkoh/SXT images of the flare spanning the period shown in Figure 1. Each image is an average of full-resolution flare-mode images taken through the AlMg or Al12 filters for the period shown in each panel, intended to demonstrate the morphological changes that occurred. Contours are at $5,15,25, \ldots, 95 \%$ of the maximum in each panel. Overplotted on each panel (in dashed black and white lines) are the morphology of the radio loop at the same period obtained as described in the text. The times of these loops are 03:08:21, 03:11:01, 03:13:26, 03:16:16, 03:17:46 and 03:20:01 UT. 


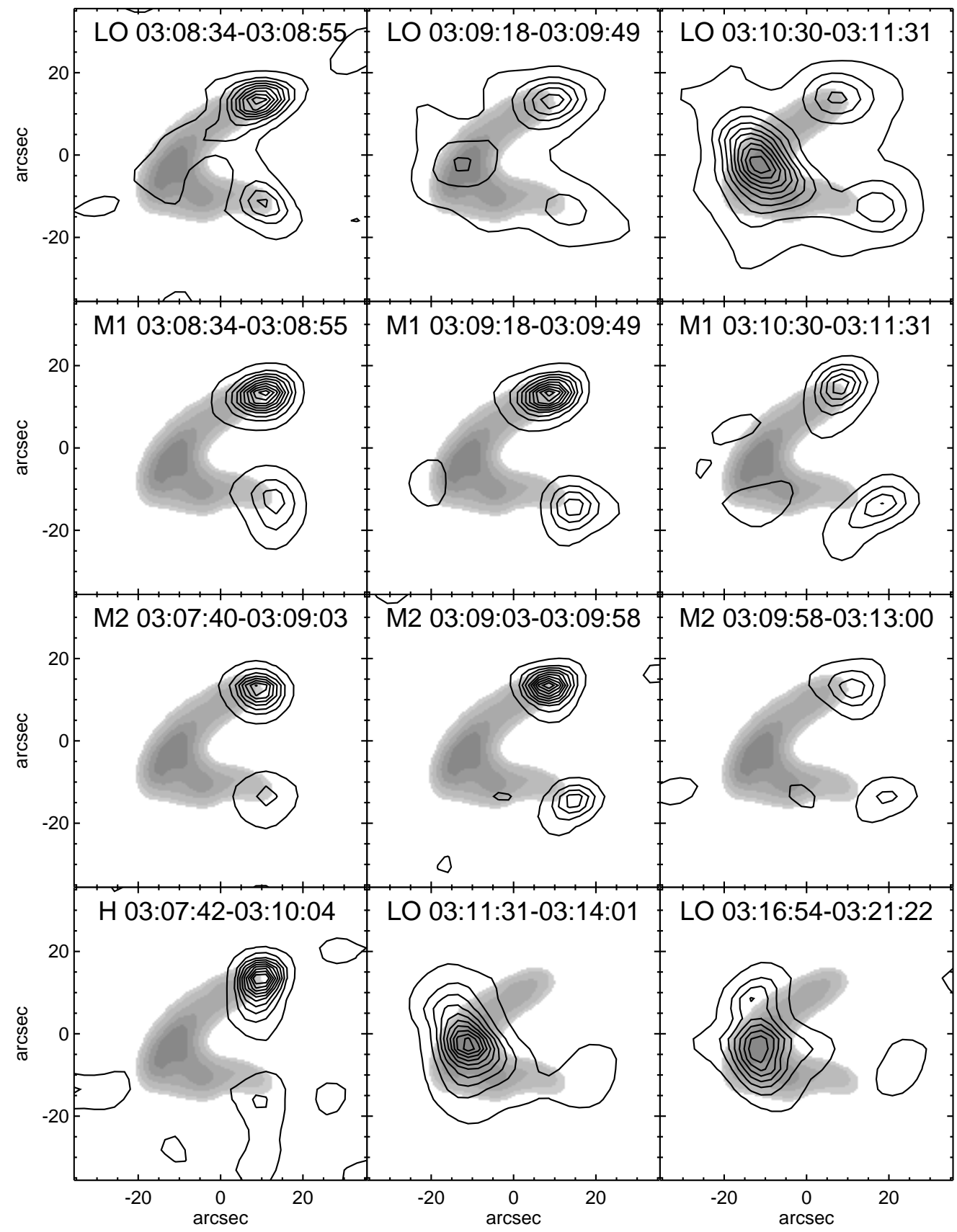

Fig. 6.- Yohkoh/HXT hard X-ray images (contours) overlaid on an SXT image (greyscale representation of the period 03:11:00 - 03:11:54, i.e. the second panel in Figure 5). The three columns essentially present three different times during the rise and peak of the radio emission. Due to the low count rate only a single image can be made in the highest energy channel $(\mathrm{H})$, shown in the lower left panel; the remaining panels on the bottom row of the figure are $\mathrm{LO}$ channel images from times after 03:12 UT when there are essentially no counts in the M1, M2 and $\mathrm{H}$ channels. Contour intervals are at $5,15, \ldots, 95 \%$ of $7,6,7,2$ counts $\mathrm{cm}^{-2}$ for the LO, M1, M2, H images, respectively, except for the two LO images in the lower row where the contours are percentages of 25 counts $\mathrm{cm}^{-2}$. 


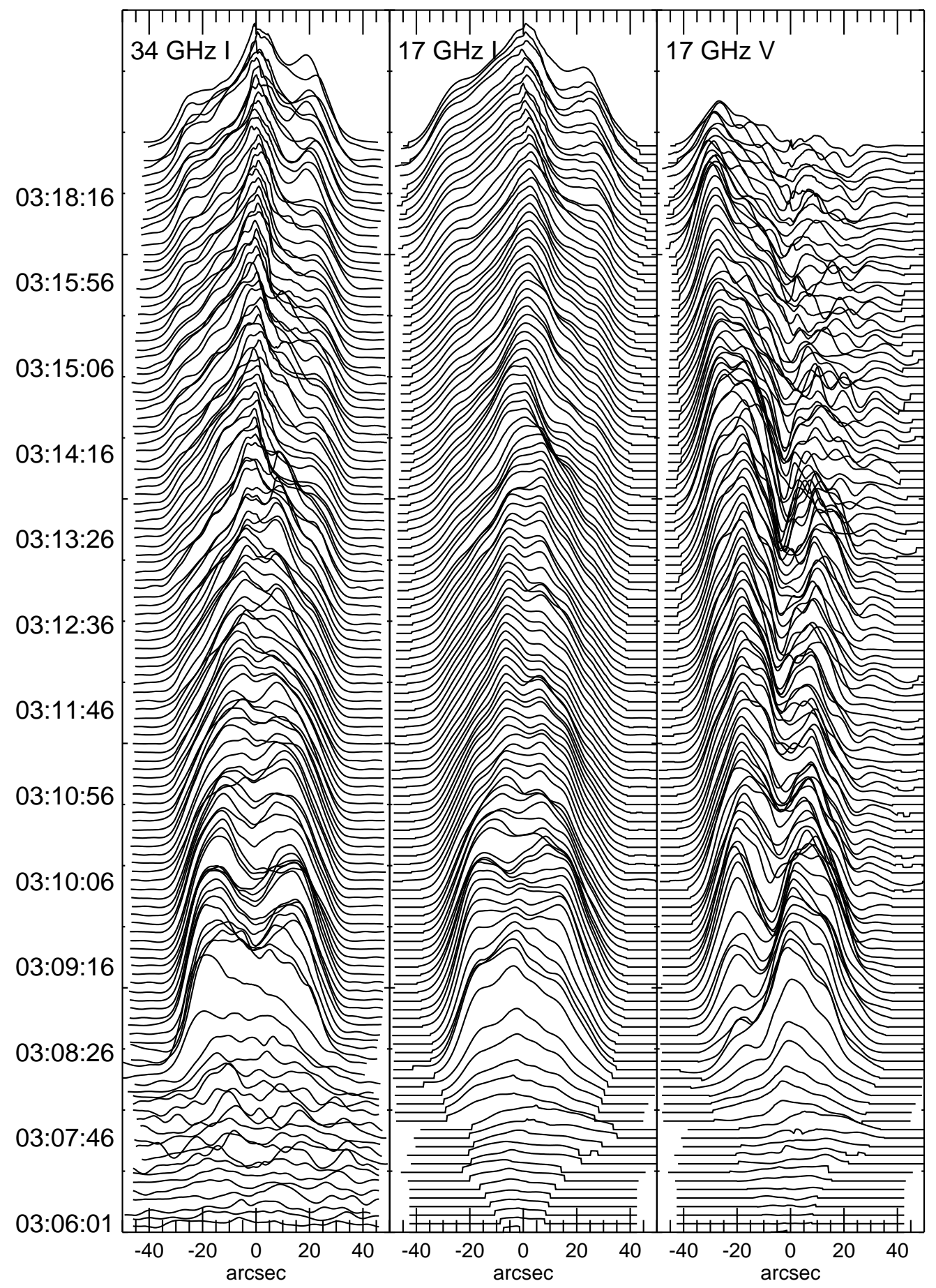

Fig. 7.- Intensity profiles measured along the loop for each of 128 maps at $34 \mathrm{GHz}$ (left column), $17 \mathrm{GHz}$ I (middle column) and $17 \mathrm{GHz} \mathrm{V}$ (i.e., circularly-polarized flux; right column). The times run from 03:06:01 UT at the bottom of each column to 03:20:01 at the top, with intervals between images being irregular but generally $5 \mathrm{~s}$, with a longer separation of $15 \mathrm{~s}$ for the first 7 and last 17 images. The left side of each profile corresponds to the southern leg of the loop. The profiles are scaled to the maximum at each time provided it exceeds either $2 \times 10^{5} \mathrm{~K}(34 \mathrm{GHz})$ or $5 \times 10^{5} \mathrm{~K}$ $(17 \mathrm{GHz} \mathrm{I}$ and $\mathrm{V})$. 


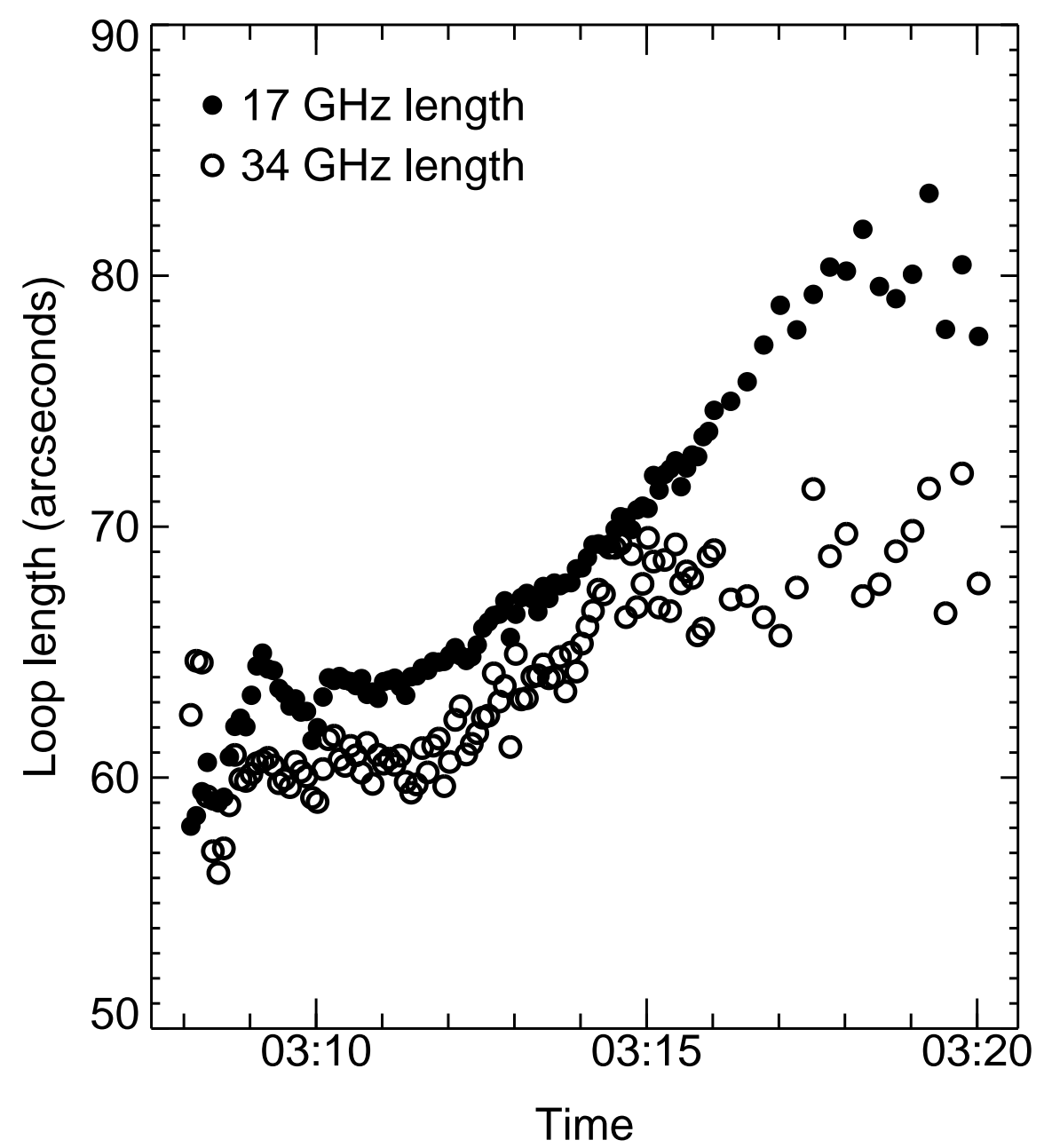

Fig. 8.- Plot of the variation of the loop length with time, where the length is defined to be the distance measured along the "spine" of the loop between the extrema at $10 \%$ of the maximum intensity for each time (see Figure 8). Data are plotted for both $17 \mathrm{GHz}$ (filled circles) and $34 \mathrm{GHz}$ (open circles). 


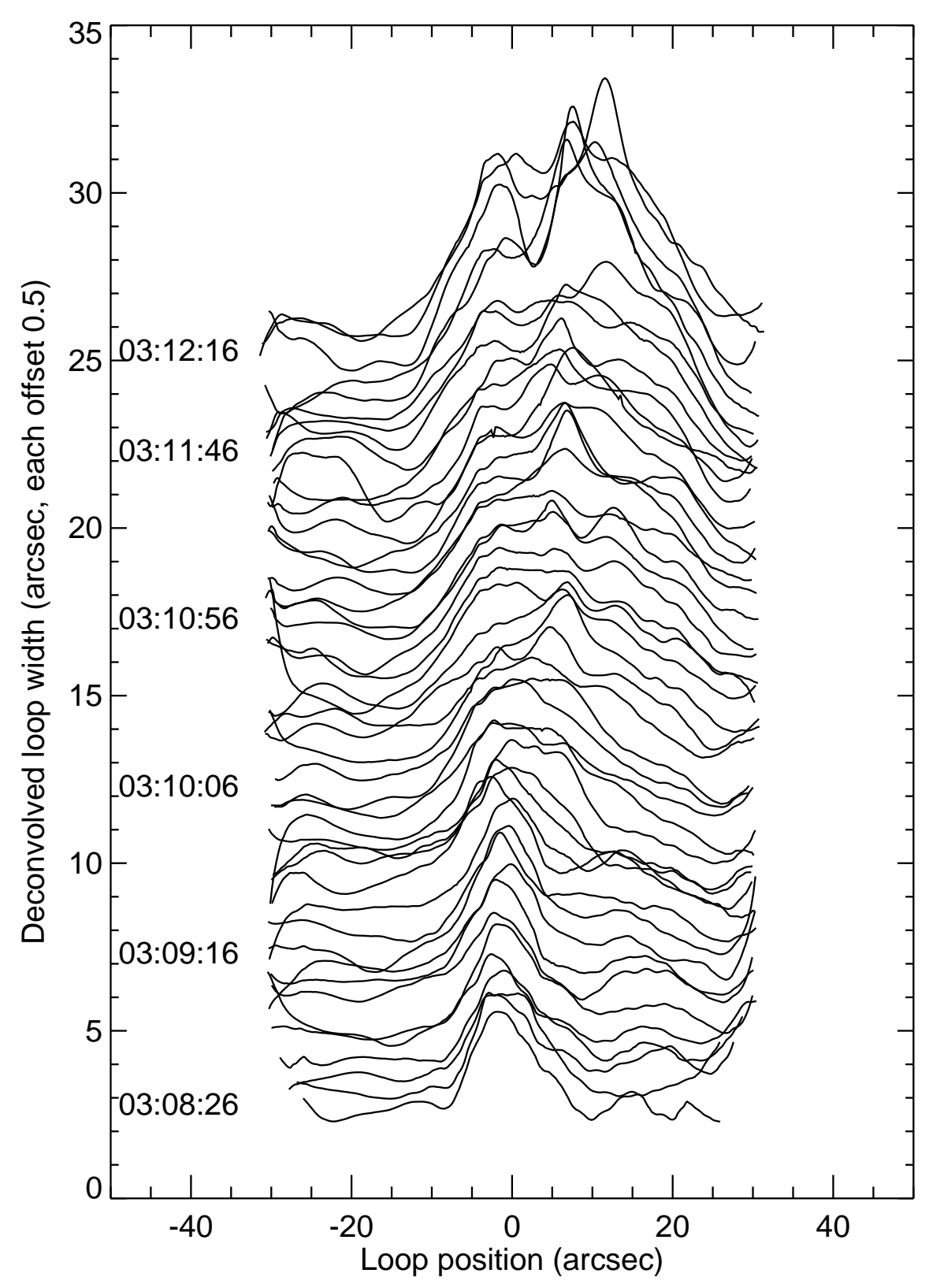

Fig. 9.- Measurements of the deconvolved width of the loop (measured by fitting a Gaussian profile to a cross-section taken orthogonal to the loop direction at each point) as a function of distance along the loop for $34 \mathrm{GHz}$ images between 03:08:26 and 03:12:16. Subsequent profiles are offset by $0 . " 5$ from the preceding profile, so the upper profile is offset by $22 . " 5$ from the first profile. The profiles generally show loop widths of 2-3" except at the top of the loop where the apparent thickness tends to be larger, at least partly because of the curvature of the loop there. The widths are full width at half maximum. 

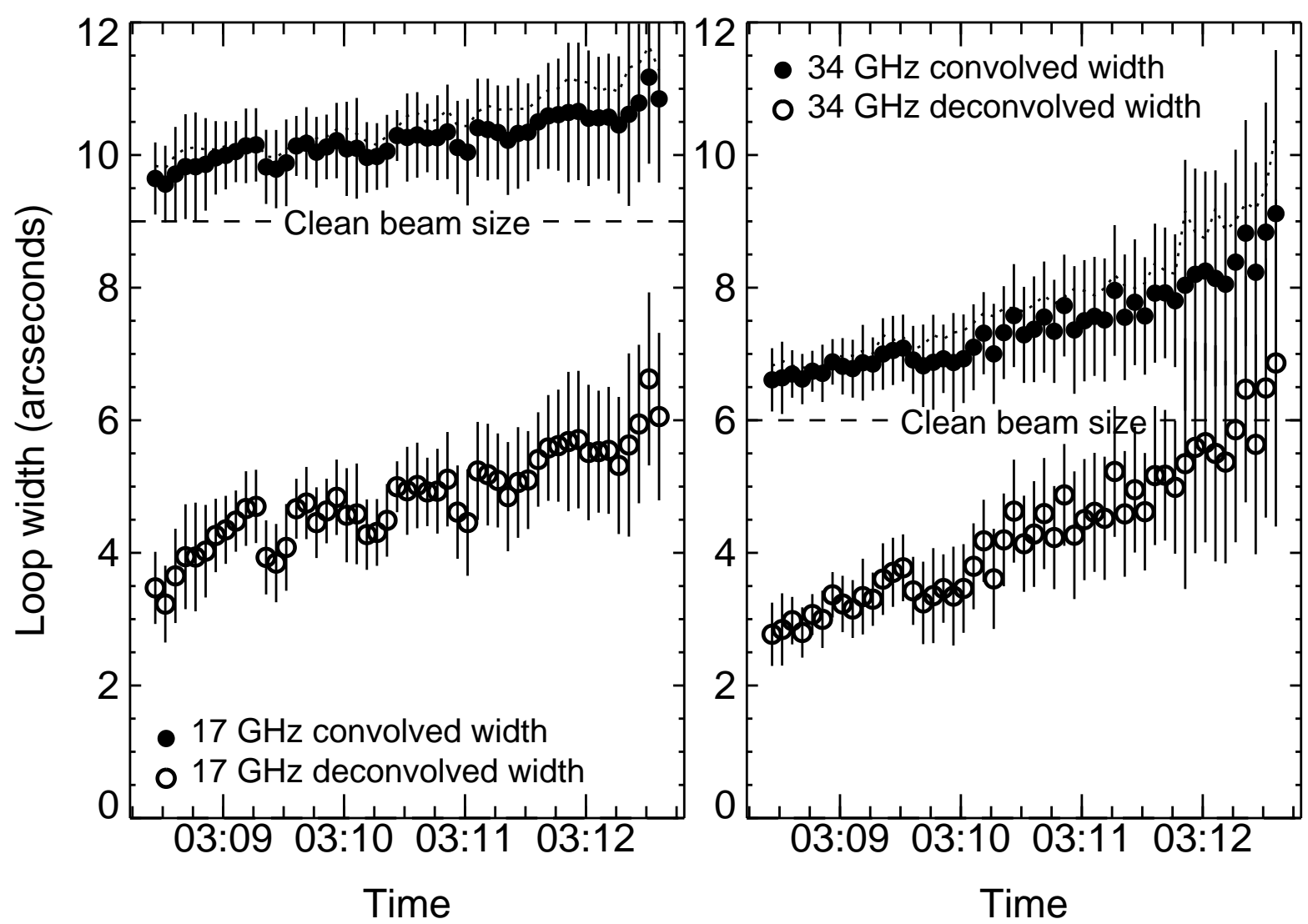

Fig. 10. - Plots of the variation in the median loop width as a function of time at both 17 and $34 \mathrm{GHz}$. The plotted quantity is the median width measured at each point along the loop (i.e. the median values of the data shown in Figure 9), while the error bars are the standard deviation of the measurements weighted by the intensity at each point. The deconvolved value is obtained by removing the effects of the beam size in each image from the measurement. The quantity plotted is the full width at half maximum. 


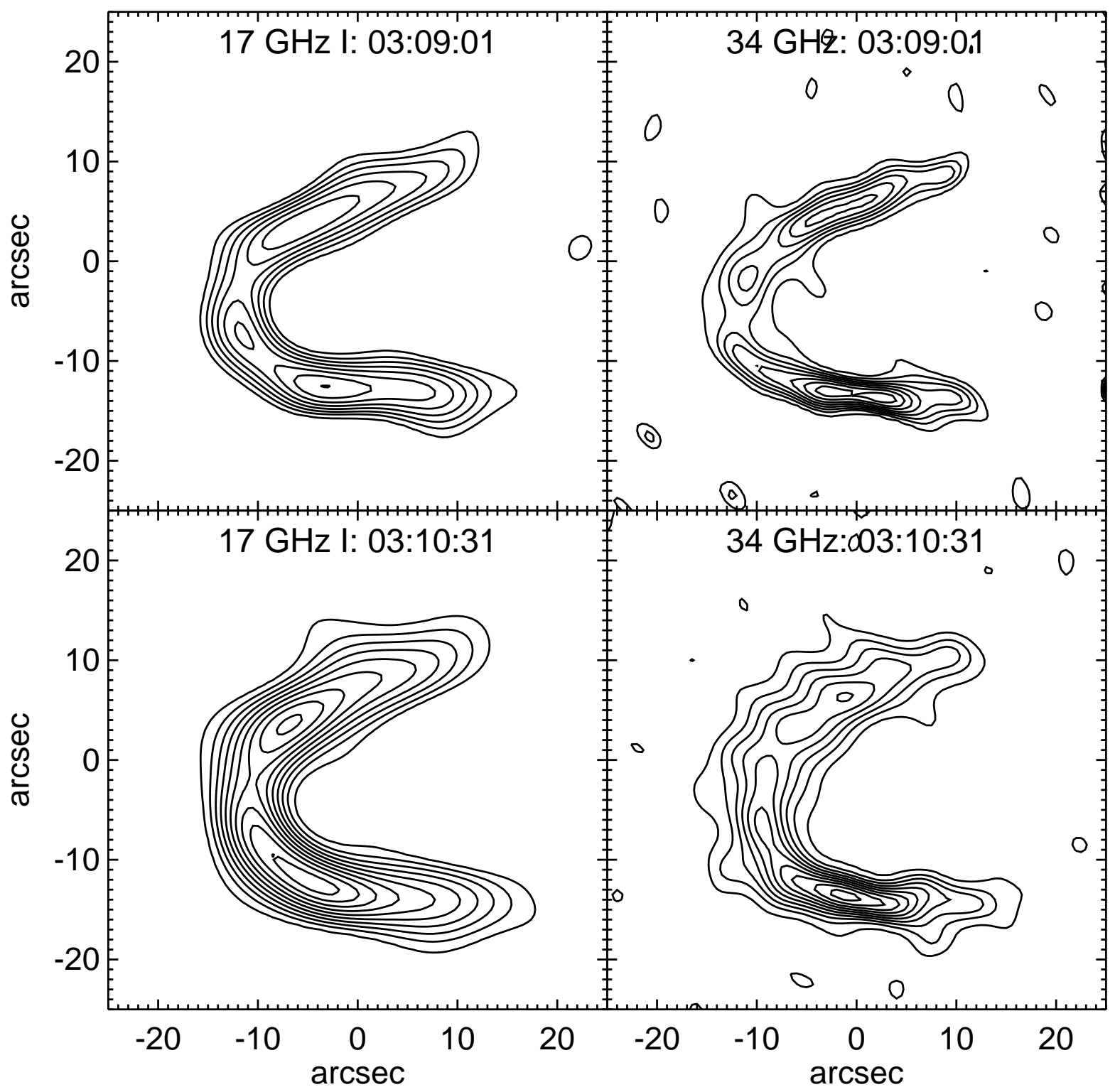

Fig. 11.- 17 and $34 \mathrm{GHz}$ NoRH images at two selected times deconvolved using 0.'5 pixels and a maximum entropy algorithm implementing a smoothness constraint. These are the model brightness distributions resulting from the deconvolution, i.e., they have not been smoothed with a gaussian in the way that the images in Figure 4 were. In these images we effectively trade off dynamic range for spatial resolution. At this resolution the $17 \mathrm{GHz}$ circular polarization images show little significant emission. The effective spatial resolution varies with position in these images depending on the local signal-to-noise; the narrowest features visible have a FWHM of order $3^{\prime \prime}$. Contours are plotted at $5,15,25, \ldots, 95 \%$ of the peak in each image $\left(2.8\right.$ and $4.0 \times 10^{7} \mathrm{~K}$ at 17 $\mathrm{GHz}, 1.1$ and $1.5 \times 10^{7} \mathrm{~K}$ at $34 \mathrm{GHz}$ ). 


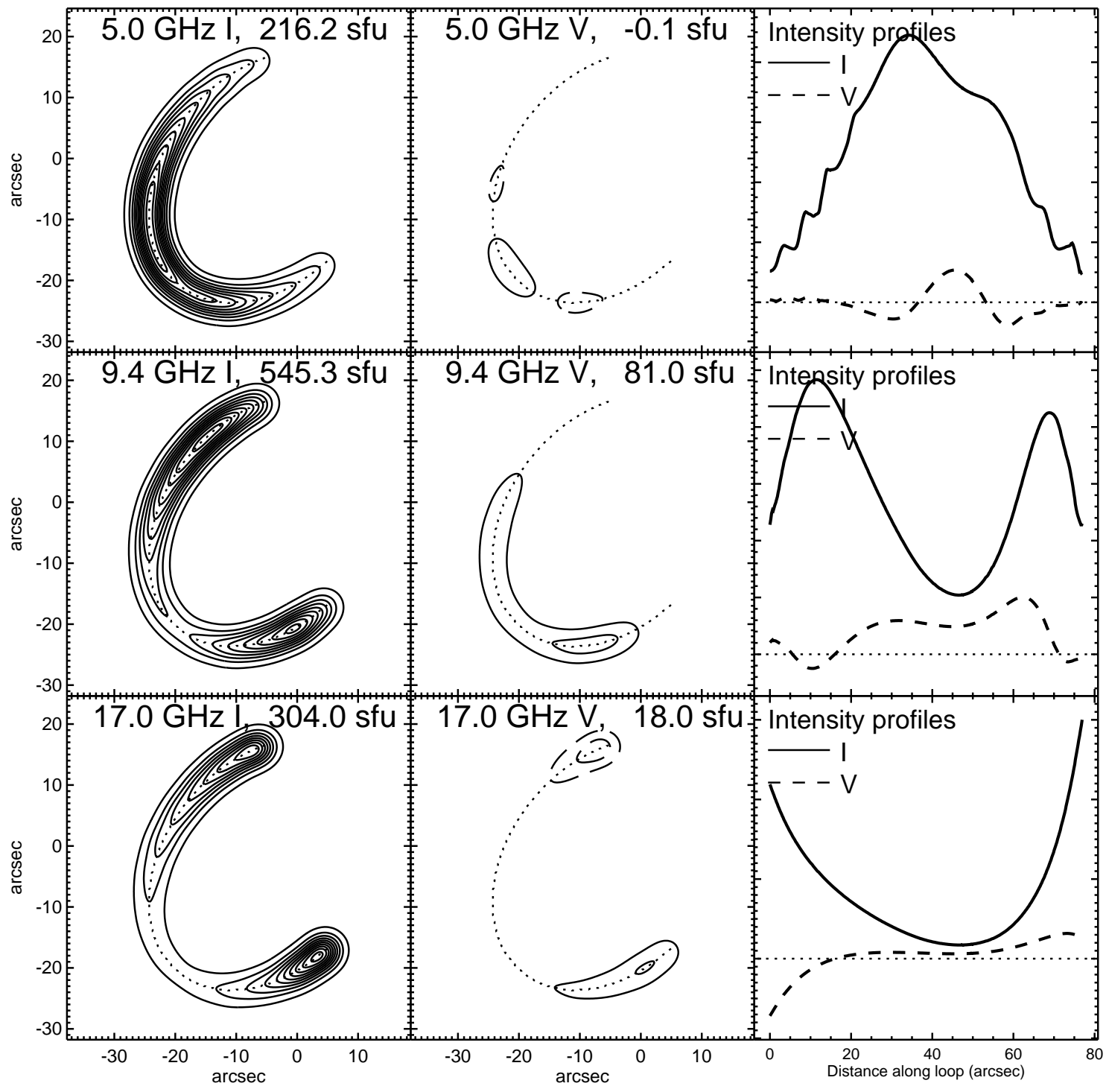

Fig. 12.- Models of the appearance of a flare loop at an optically-thick low frequency $(5.0 \mathrm{GHz}$, top row), a frequency near the peak in the radio spectrum $(9.5 \mathrm{GHz}$, second row) and an optically thin higher frequency $(17.0 \mathrm{GHz})$. The three columns show (left) the total intensity image, (middle) the circularly polarized image, and (right) flux profiles plotted versus distance measured along the loop. The loop is filled with gyrosynchrotron-emitting nonthermal electrons with an energy spectral index of -3.5, a footpoint magnetic field of $600 \mathrm{G}, 10^{8}$ electrons above $10 \mathrm{keV}$, a loop height of $28^{\prime \prime}$ and thickness at the looptop of $2^{\prime \prime}$ and a footpoint separation of $44^{\prime \prime}$. The pitch angle distribution is uniform throughout the loop, corresponding to a relaxed distribution and implying a uniform particle density as well. The loop is located at a longitude of $70^{\circ}$ east, latitude $30^{\circ}$ south and is turned at an angle of $60^{\circ}$ to the equator, so that the apparent footpoint separation is much smaller. The magnetic field at the looptop is $224 \mathrm{G}$. The dotted line indicates the location of the spine of the loop; contours are at $5,15, \ldots, 95 \%$ of the peak in the total intensity map at each frequency. The morphology of the loop does not change with frequency as long as the loop is optically thin, so the $17 \mathrm{GHz}$ appearance is representative of all high frequencies. 